\title{
Self-Similar Solutions of the G-Equation - Analytic Description of the Flame Surface
}

\section{Barna IF*}

Wigner Research Center of the Hungarian Academy of Sciences Konkoly-Thege út 29-33, 1121 Budapest, Hungary

\begin{abstract}
The main feature of the flame kinematics can be described with the G-equation. We investigate the solutions of the G-equation with the well-known self-similar Ansatz. The results are discussed and the method how to get selfsimilar solutions is briefly mentioned.
\end{abstract}

Keywords: Flame kinematics; Combustion engineering; Fluid dynamics

\section{Introduction}

To understand the dynamics of flame fronts is a general interest for combustion engineering. One way to gain such Knowledge is the investigation of the G-equation model. Since the G-equation was introduced by Markstein in 1964 [1] numerous analytical [2] and numerical approaches [3-5] have been used to find the solutions. The study of [2] uses the method of characteristics for nonlinear first order partial-differential equations (PDA).

Dekena [5] uses a computational fluid dynamics (CFD) code Fire to investigate turbulent flame propagation in Gasoline Direct Injection engines. Further references for application of the G-equation are given therein.

In the G-equation model the flame itself is treated as a surface (flame front) that separates the burnt from the unburnt gas. A detailed derivation of the model can be found in ref. [2]. The flame front is described here by the relation of:

$G(r, z, t)=z-\zeta(r, t)=0$

The most general form of the G-equation is the following:

$$
\frac{\partial \zeta(r, t)}{\partial t}+u \frac{\partial \zeta(r, t)}{\partial r}-v+S_{l} \sqrt{1+\left(\frac{\partial \zeta(r, t)}{\partial r}\right)^{2}}=0
$$

Where $u(r, z, t)$ and $v(r, z, t)$ are the radial and the axial components of the gas velocity, and $S_{L}$ is the constant modulus of the laminar burning velocity, respectively.

In the following we use a well-known method, which is very popular to investigate nonlinear PDAs, namely searching for selfsimilar solutions. We are looking for solution of (2) in the form of:

$$
\zeta(r, t)=t^{-\alpha} f\left(\frac{r}{t^{\beta}}\right):=t^{-\alpha} f(\eta)
$$

The similarity exponents $\alpha$ and $\beta$ are of primary physical importance since $\alpha$ represents the rate of decay of the magnitude $\zeta(x, t)$, while $\beta$ is the rate of spread (or contraction if $\beta<0$ ) of the space distribution as time goes on.

This kind of solutions has a broad range of applicability, e.g. a large number of diffusion-reaction-type equations [6] or time-dependent telegraph-type heat-conduction equation [7] can be examined as well.

Substituting this into (2) we have:

$$
\begin{aligned}
& -\alpha t^{-\alpha-1} f(\eta)-\beta t^{-\alpha-\beta-1} f^{\prime}(\eta) r-u(r, z, t) t^{-\alpha-\beta} f^{\prime}(\eta)-u(r, z, t)+ \\
& S_{l} \sqrt{1+t^{-2 \alpha-2 \beta}\left[f^{\prime}(\eta)\right]^{2}}=0
\end{aligned}
$$

Where prime denotes differentiation with respect to $\eta$.

After setting set $u=S_{l}=1$ and $v=0$, one can see that this is a nonlinear ordinary differential equation (ODE) if and only if $\alpha=-1$ and $\beta=1$ (the universality relation). The corresponding ODE we shall deal with is:

$$
f(\eta)_{1}=c_{1} \eta-c_{1}-\sqrt{1+c_{1}^{2}} ; \quad f(\eta)_{2}=c_{1} \sqrt{\eta(\eta-2)}
$$

The solutions can be easily obtained and read:

$$
f(\eta)_{1}=c_{1} \eta-c_{1}-\sqrt{1+c_{1}^{2}} ; \quad f(\eta)_{2}=c_{1} \sqrt{\eta(\eta-2)}
$$

Where $c_{1}$ is the real integration constant. The solutions can be seen in Figure 1. The corresponding self-similar solutions are:

$$
\zeta(r, t)_{1}=c_{1} r-c_{1}-\sqrt{1+c_{1}^{2}} ; \quad \zeta(r, t)_{2}=c_{1} t \sqrt{\left(\frac{r(r-2 t)}{t^{2}}\right)}
$$

Both solutions can be proven with direct derivation. The first solution is trivial however the non-trivial second one is presented on Figure 2.

We may consider more sophisticated flow systems where the radial and axial components of the gas velocity and the $S_{L}$ are functions of time and radial position. First let's consider that the velocities and the constant modulus of the laminar burning velocity are real numbers $(u$, $v, S_{l} \in \mathfrak{R}$.) Now the ODE has the following form:

$$
f(\eta)-\eta f^{\prime}(\eta)-f^{\prime}(\eta)-v+\sqrt{1+\left[f^{\prime}(\eta)^{2}\right]}=0
$$

The solutions became a bit more complicated:

$$
f(\eta)_{1}=c_{1} \eta+u c_{1}+u-S_{l} \sqrt{1+c_{1}^{2}} ; f(\eta)_{2}=c_{1} \sqrt{-u^{2}-\eta^{2}+S_{L}^{2}-2 \eta u}+v
$$

*Corresponding author: Barna IF, Wigner Research Center of the Hungarian Academy of Sciences Konkoly, Thege út 29-33, 1121 Budapest, Hungary, Tel: +36-1-392-2222/3504; Fax: +36-1-392-2598; E-mail: barna.imre@wigner.mta.hu

Received January 19, 2017; Accepted July 05, 2017; Published July 10, 2017

Citation: Barna IF (2017) Self-Similar Solutions of the G-Equation - Analytic Description of the Flame Surface. J Generalized Lie Theory Appl 11: 274. doi: 10.4172/1736-4337.1000274

Copyright: (c) 2017 Barna IF. This is an open-access article distributed under the terms of the Creative Commons Attribution License, which permits unrestricted use, distribution, and reproduction in any medium, provided the original author and source are credited. 


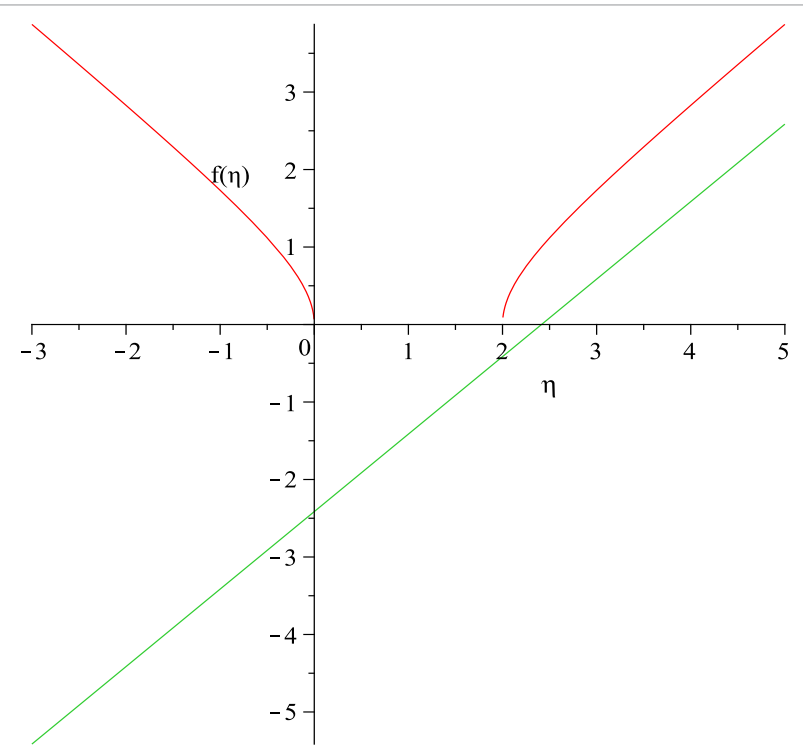

Figure 1: Solutions of eqn. (5), red line presents $f(\eta)_{2}$ and the green one shows $f(\eta)_{1}$.

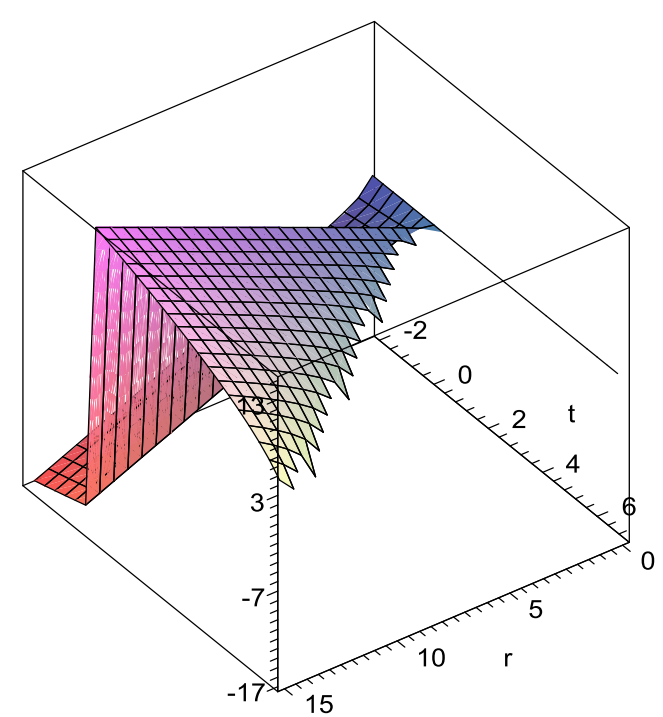

Figure 2: The self-similar solution $\zeta(r, t)_{2}$, from eqn. (7) in the range $r=0 . .15$, $t=-2 . .7$ for $c_{1}=1$.

The function $f(\eta)_{2}$ is presented in Figure 3. with the following fixed set of parameters $\left(u=2, v=-1, c_{1}=1, S_{L}=3\right)$.

Note, that the solution has now a compact support with nonvanishing first derivatives at the border. This means that there is no fluxconservation at the boundaries. There are more analytical solutions for special $u(x, t), v(x, t)$ and $S_{l}(x, t)$ functions. The only remaining task is to give reasonable physical interpretation for $u(x, t), v(x, t)$ and $S_{l}(x, t)$.

Another generally interesting question is the dispersion relation and the attenuation distances. It can be examined how wave equations or other nonlinear evolutionary equations propagate plain waves in time and in space. Inserting the usual plain wave approximation $\zeta(r, t)=e^{i(k r+\omega t)}$ into eqn. (2) the dispersion relation and the attenuation distance can be obtained. These are the followings:

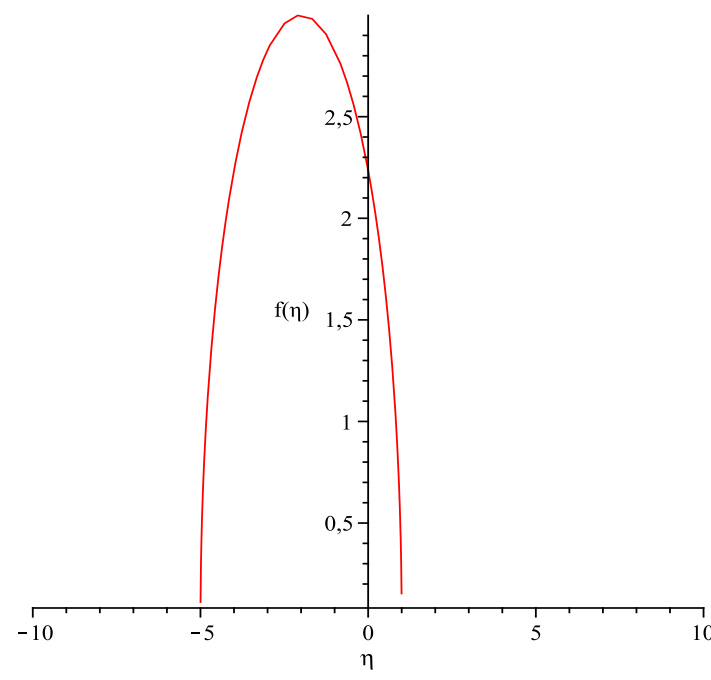

Figure 3: The solution $f(\eta)_{2}, u=2, v=-1, c_{1}=1, S_{L}=3$

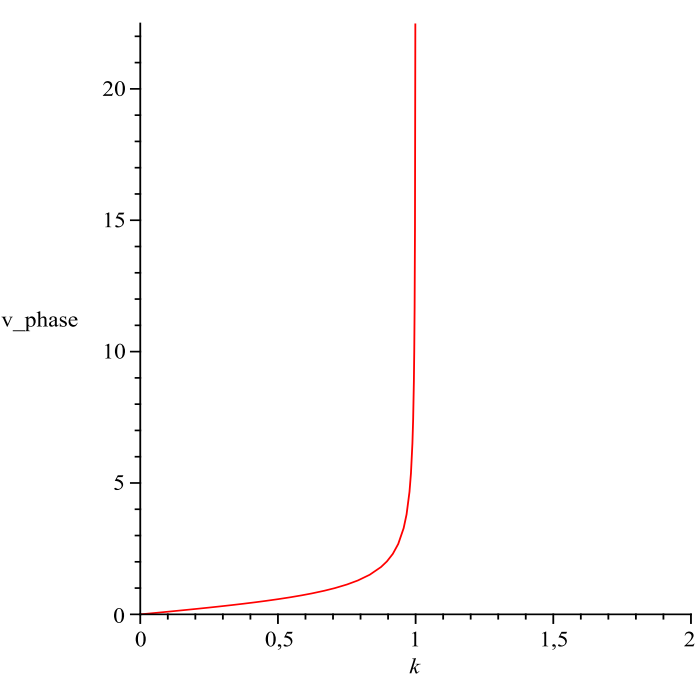

Figure 4: The dispersion relation $v_{p}(k)$ for eqn. (2).

$$
\begin{aligned}
& v p=\frac{\omega}{\operatorname{Re}(k)}=\frac{\omega}{\frac{1}{2} \sqrt{\left|-1+k^{2}\right|}\left(1-\operatorname{signum}\left[-1+k^{2}\right]\right)} ; \\
& \alpha=\frac{1}{\operatorname{Im}(k)}=\frac{1}{\omega+k+\frac{1}{2} \sqrt{\left|-1+k^{2}\right|}\left(1-\operatorname{signum}\left[-1+k^{2}\right]\right)}
\end{aligned}
$$

Inserting the relation of $\omega=k c$ where $c$ is the propagation velocity of the signal the formulas only depend on the wavenumber vector $k$. Considering $c=1$ propagation speed Figure 4 shows the phase velocity as the function of the wavenumber. The $\left(1-\operatorname{signum}\left[-1+k^{2}\right]\right)$ in the formula is responsible for the compact support of the function. Figure 5 shows the attenuation distance of the various waves. At $k=1$ the $\alpha(k)$ function is non-analytic.

\section{Conclusion}

In this short study we just wanted to present that self-similar solution can be easily used to generate analytic solutions for the G-equation. With more general and more physical relations for the radial and axial 


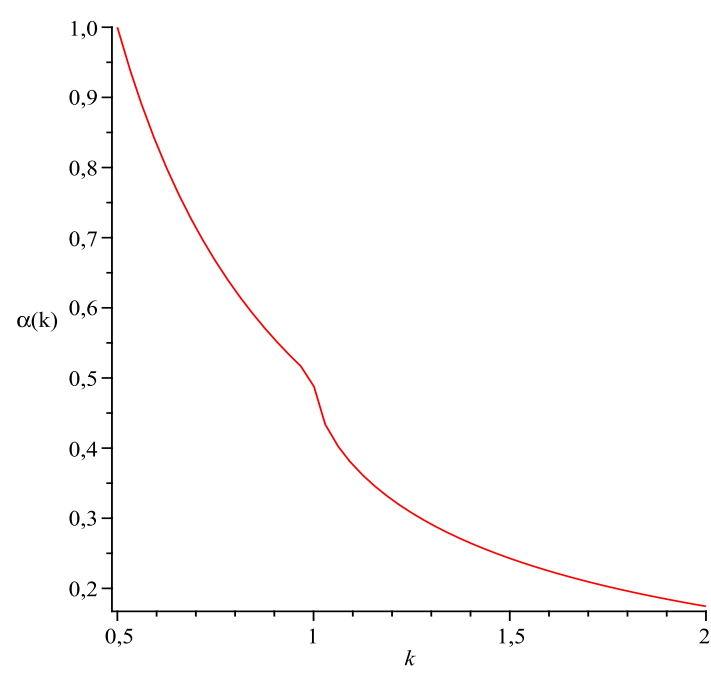

Figure 5: The attenuation function ( $k$ ) for eqn. (2).

gas flow velocity hopefully more physical solutions can be obtained. Anyhow, any kind of analytical solution of a nonlinear PDA can be useful giving a solid basis for testing complex and sophisticated two or three dimensional numerical finite-element computational fluid dynamics codes.

\section{References}

1. Markstein GH (1964) Non steady flame propagation Pergamon Press, Oxford.

2. Bondar ML, Mattheij RMM, ten Thije JHM (2007) Investigation of Bunsen flame dynamics by the method of characteristics. IMA Journal of Applied Mathematics 72: 405-419.

3. Pitsch H (2002) A G-equation formulation for large-eddy simulation of premixed turbulent combustion. Center for Turbulence Research Annual Research Briefs.

4. Liang L, Reitz RD, Yi J, lyer CO (2006) A G-equation Combustion Mode Incorporating Detailed Chemical Kinetics for PFI/DI SI Engine Simulations. Sixteenth International Multidimensional Engine Modeling User's Group Meeting at the SAE Congress.

5. Dekena M, Peters N (1999) Combustion Modeling with the G-Equation. Oil \& Gas Science and Technology Rev. IFP 54: 265-270.

6. Gilding BH, Kersner R (2004) Travelling Waves in Nonlinear DiffusionConvection Reactions. Progress in Nonlinear Differential Equations and Their Applications, Birkhauser Verlag, Basel-Boston-Berlin, ISBN 3-7643-7071-8.

7. Barna IF, Kersner R (2010) Heat conduction: a telegraph-type model with selfsimilar behavior of solutions. J Phys A Math Theor 43: 375210. 